\title{
Implantação do modulo de Gestão de Configuração do framework ITIL: um estudo de caso
}

\section{Title: Implementation of ITIL Configuration Management module: a case study}

\author{
Fernando Couto, Lucila Ishitani
}

Instituto de Ciências Exatas e Informática (ICEI) - Pontifícia Universidade Católica de Minas Gerais (PUC Minas) - 30640070 - Belo Horizonte - MG - Brasil

$$
\text { f.couto@live.com, lucila@pucminas.br }
$$

\begin{abstract}
This paper is based on the operation of one of the most used IT service management models by organizations around the world, ITIL. With the purpose of expanding knowledge in the area, the main proposal of this article is to present experiences, viewpoints, criticism and added value generated during and after the implementation of the ITIL Configuration Management module in a Brazilian public agency. As a successful case, its main objective is to measure the direct and indirect user's satisfaction level of the proposed module, in order to explore the main benefits of an implementation based on best practice and, to propose improvements and suggestions for future implementation processes.
\end{abstract}

Keywords. CMDB; ITIL; ITSM; Transition; Configuration Management; Impact Analysis; IT Management.

Resumo. Este trabalho se baseia no funcionamento de um dos modelos de gestão de serviços de TI mais utilizados pelas organizações de todo o mundo, o ITIL. Com o objetivo de expandir o conhecimento na área, a principal proposta deste artigo é expor experiências, pontos de vistas, críticas e valor agregado gerados durante e após a implantação do módulo de Gestão da Configuração da ITIL em um órgão público brasileiro. Por ser um caso de sucesso, o principal objetivo é mensurar o nível de satisfação do usuário direto e indireto do módulo em questão, explorando os principais benefícios de uma implantação baseada nas melhores práticas do campo, além de propor melhorias e sugestões aos processos de implantação futuros.

Palavras-Chave. CMDB; ITIL; ITSM; Transição; Gestão de Configuração; Análise de Impacto.

\section{Introdução}

Independentemente do tamanho, tipo ou ramo de uma empresa, a informação e o conhecimento são elementos do negócio que têm recebido bastante atenção de suas gerências. Isso porque a forma como estes são tratados e disponibilizados pode influenciar diretamente a eficiência e eficácia de tomadas de decisão, resolução de problemas, análises de impacto, previsões de mudança, entre outras tarefas [Esteves 2014].

Cite as: $\quad$ Couto, F., Ishitani, L. (2018). Implementation of ITIL Configuration Management module: a case study (Implantação do modulo de Gestão de Configuração do framework ITIL: um estudo de caso). iSys: Revista Brasileira de Sistemas de Informação (Brazilian Journal of Information Systems), 11(3), 70-89. 
$\mathrm{Na}$ área de Tecnologia da Informação, a Ciência dos Serviços tem buscado, incessantemente, o aprimoramento das operações, entrega e suporte de TI (Tecnologia da Informação). Para este propósito, surgiu o Gerenciamento de Serviços de TI (GSTI ou ITSM, do inglês IT Service Management) que, por sua vez, é definido como uma abordagem para operações de TI caracterizada por sua ênfase em serviços, consumidores, acordo de níveis de serviço (ANS ou SLA, do inglês, Service Level Agreement) e a manipulação de funções de TI em atividades diárias por meio de processos [Conger et al. 2008].

No contexto de ITSM, encontra-se a ITIL - Biblioteca de Infraestrutura de Tecnologia da Informação ou, do inglês, Information Technology Infrastructure Library - como um dos modelos mais utilizados no mundo por empresas que procuram reduzir custos, melhorar a segurança e elevar o nível dos serviços de TI [Batmunkh et al. 2014].

Este trabalho revisou as principais disciplinas do framework ITIL, focando principalmente na Gestão da Configuração (GC), um dos módulos da disciplina de Transição de Serviços, que tem por objetivo sugerir as melhores práticas perante dificuldades comuns que empresas enfrentam enquanto organizam seus ativos de infraestrutura de TI [Lema et al. 2015]. Esta tarefa, inicialmente, pode ser trabalhosa e requerer atenção de cada uma das partes interessadas, uma vez que envolve modelagens de mapeamento de ativos de todo o ambiente de tecnologia da organização.

Ao citar a GC, sob uma perspectiva ITIL, é necessário o entendimento do conceito de CMDB (Banco de Dados de Gestão da Configuração, ou do inglês, Configuration Management Database), já que é a base que garante o bom funcionamento dos outros processos do ITIL. Tal termo nasceu das melhores práticas ITIL e refere-se a um software capaz de unificar e armazenar dados e informações sobre ativos de TI que, neste contexto, são chamados de CIs (Item de Configuração, ou do inglês, Configuration Item) [Yamada et al. 2013].

Um software CMDB deve ir muito além do que apenas armazenar informações sobre configurações de ativos. Ele também deve ser capaz de armazenar suas dependências e relacionamentos entre esses ativos. De outro ponto de vista, um CMDB é um cubo n-dimensional de todo o ambiente de TI, incluindo históricos de relacionamentos entre os CIs, e deve ser mantido atualizado para que o planejamento de mudanças reflita a realidade do ambiente. Caso esta base não reflita a realidade dos relacionamentos de ativos de TI da organização, o CMDB será mais uma grande responsabilidade do que uma vantagem [Marquis 2007].

Diante deste cenário, e baseado nos resultados deste trabalho, uma das etapas mais desafiadoras de uma implantação de GC é a contextualização de seus conceitos, os quais devem ser bem trabalhados com as partes envolvidas. Neste caso, é importante que o apoio da gerência da organização seja visível e claro, guiando cada integrante envolvido ao objetivo final do projeto: a melhoria da prestação dos serviços de TI.

\subsection{Objetivos}

A realização deste estudo teve como principal objetivo acompanhar e retratar, do ponto de vista crítico e analítico, a implantação do módulo de Gestão da Configuração no setor de tecnologia de um órgão público do Brasil. Além disso, foi possível a análise do estado do negócio, anterior e posteriormente à implantação, a validação do trabalho realizado

isys: Revista Brasileira de Sistemas de Informação (isys: Brazilian Journal of Information Systems) 
pelos profissionais com base nas melhores práticas do framework, mensurar o nível de satisfação dos usuários de TI de diferentes áreas do setor e, enfim, identificar os maiores ganhos e/ou perdas do projeto.

Como contribuição inédita ao meio científico, este trabalho também visou proporcionar um alto nível de detalhes de cada etapa executada em uma empresa de grande porte, além de mensurar o sentimento do usuário final em relação à nova maneira de gerir os itens de configuração da organização.

\subsection{Motivação}

A organização e mapeamento dos serviços e ativos de TI corroboram para que seus serviços funcionem eficientemente. Porém, é importante que a decisão de organizá-los e mapeá-los tenha apoio direto da gerência da empresa [Bayona et al. 2017].

No contexto deste trabalho, o apoio da alta gerência pela evolução dos processos ITIL dentro da organização passou a ser visível e ativo, uma vez que as partes interessadas perceberam que a desorganização e falta de mapeamento dos ativos e serviços de TI estavam gerando atraso de atendimento, informações não confiáveis e até mesmo certa dificuldade em realizar análises de impacto antes que uma mudança no ambiente de TI ocorresse.

O órgão já possuía softwares capazes de entregar este produto necessário, porém, ainda não os utilizavam com eficácia. A partir disso, a Diretoria de Informática decidiu contratar uma consultoria para que estas ferramentas trabalhassem em conjunto, agregando valor aos seus processos de TI.

\section{Uma visão do campo}

Segundo Orta e outros (2014), "ITSM refere-se à implementação e gerenciamento da qualidade de serviços de TI, a fim de sustentar as necessidades do negócio". Para que o conceito ITSM se encaixe nas operações de TI, organizações internacionais propõem conjuntos de melhores práticas e padrões de gerenciamento, mais conhecidos como frameworks, com o objetivo de facilitar sua implementação e a gestão destas operações ou atividades [Iden e Eikebrokk 2013]. Entre os principais frameworks conhecidos no mercado mundial, estão: ITIL v3, ISO 20.000-4 e COBIT 4.0 [Mora et al. 2014].

\subsection{O framework ITIL}

Apesar de a quantidade exata de organizações que adotam o framework ITIL ser desconhecida, existem muitos indicadores de sua expansão e adesão [Marrone e Kolbe 2014]. Um desses indicadores é o aumento do interesse ao tema em literatura científica, como é o caso deste trabalho [Vlietland e Vliet 2015].

Atualmente, as melhores práticas ITIL estão detalhadas em cinco livros ou disciplinas da AXELOS, organização responsável por seu desenvolvimento e manutenção, sendo eles: Estratégia de Serviço, Desenho de Serviço, Transição de Serviço, Operação de Serviço e Melhoria de Serviço Continuada [Axelos Website 2018]. A Figura 1 mostra a interação entre essas cinco disciplinas. 


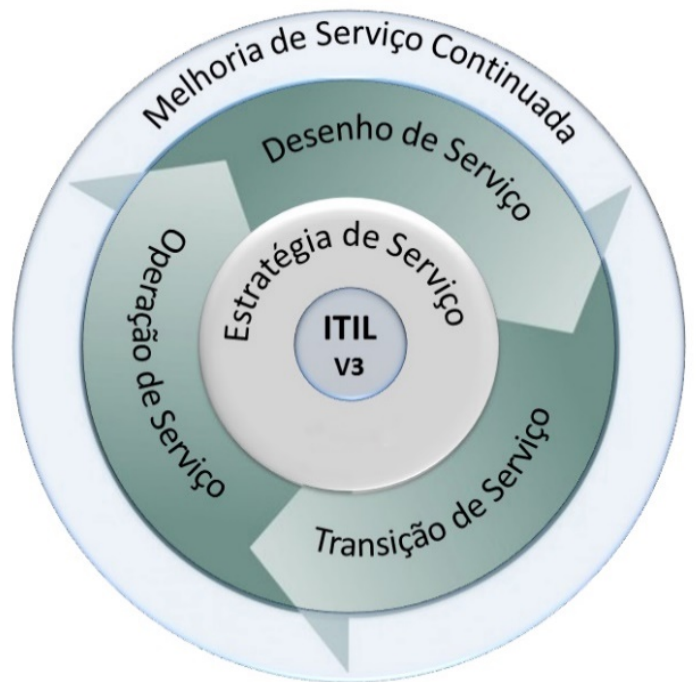

Figura 1. Ciclo de vida entre as disciplinas do framework ITIL v3 [Palma, 2013]

Entre as cinco disciplinas da ITIL, a de Transição de Serviços suporta o objeto deste trabalho: a Gestão da Configuração (GC). É nesta disciplina que é descrito como os requisitos da Estratégia, esboçados no processo Desenho, serão tratados na Operação, controlando os riscos de fracasso e impactos ao negócio. Além disso, auxilia diretamente a gestão de mudanças em processos e serviços de TI na organização [Esteves 2012].

\subsection{Gestão da Configuração: trabalhos relacionados}

No cenário acadêmico brasileiro e mundial, foram encontrados trabalhos que retratam a implementação do módulo de Gestão da Configuração do ITIL v3.

O trabalho desenvolvido por Jiang e Kamali (2008) apresentou a proposta de criação de uma disciplina especializada no assunto de GC em cursos de TI, uma vez que identificaram a ementa existente insuficiente para preparar os graduandos para o universo de GC. Antes de apresentar a proposta, os autores comentam sobre o crescimento do framework ITIL e apontam a Gestão da Configuração como o "pedaço central de todo o seu universo", acrescentando ainda que quaisquer atividades no ambiente de TI devem ser registradas e rastreadas como parte de GC. O objeto do trabalho foi o desenvolvimento de uma ementa idêntica à que era ofertada por uma universidade americana, porém com a inclusão da disciplina "Application Configuration and Management", na qual os alunos foram submetidos a ensinamentos desde o conceito de GC até uma série de exercícios em laboratórios propondo diversos casos de configuração, problemas de implementação, processos, técnicas e ferramentas. Como resultado do trabalho, os autores salientam que a nova abordagem sobre GC pareceu contribuir para o aprendizado dos alunos, apontando inclusive a dificuldade de muitos devido à falta de conhecimento em $\mathrm{GC}$ e de percepção de melhores práticas.

O trabalho de Marquis (2007) já é voltado para a parte teórica e fundamentada da Gestão de Configuração do ponto de vista original da ITIL. Nesse contexto, o autor critica empresas que têm um entendimento distorcido do que é realmente a GC, explicitando alguns pontos chaves que devem ser providos pelo CMDB, sendo eles: 1) Federação, onde o CMDB é uma base de configuração confiável e definitiva que permite a comunicação com outras bases não definitivas; 2) Reconciliação, o processo que garante 
a identidade do CI, ou seja, cada CI é único e recebe uma nomenclatura correta de acordo com seu tipo; 3) Sincronização, quando existir mais de um CMDB no ambiente. Neste caso, cada CI possui um identificador único em todas as bases. Com esse histórico de dados do CI, o CMDB deve ser capaz de descobrir mudanças no ambiente (no contexto de conteúdo ou serviço de infraestrutura), por meio de rotinas de descoberta. Logo, o sistema também será capaz de distinguir, nestes CIs, mudanças aprovadas e não aprovadas em uma organização; 4) Mapeamento e Visualização, que é uma característica que provê visualização facilitada para humanos, dos relacionamentos entre CIs.

Em sua conclusão, Marquis aponta que a confusão a respeito do conceito de GC e CMDB começa com a ideia de empresas quererem algo tangível antes de, ou sem, pensar sobre os objetivos que elas precisam atingir nesta área. Inicialmente, trabalham com a ideia de implantar uma gestão de ativos de TI e então tentam evoluí-la para um CMDB. Além disso, o autor sugere que empresas que possuem um certo nível de Gestão de Ativos, e veem a necessidade de agregar a Gestão de Configuração, utilizem de suas bases de dados em planilhas, tabelas, arquivos de modelagem, entre outras fontes, para alimentar uma ferramenta CMDB confiável.

Concordando com as afirmações de Marquis, uma das atividades trabalhadas com as equipes envolvidas - durante entrevistas e reuniões realizadas - foi a definição de conceitos sobre o módulo de GC da ITIL, o que também serviu para alinhar as ideias e expectativas das partes interessadas em relação a GC.

Esteves (2012) trabalhou em um caso de implementação de GC junto à Secretaria Regional da Cultura, Turismo e Transportes (SRT) da Região de Madeira, em Portugal. Em seu estudo, Esteves retratou a dificuldade em encontrar casos de estudo na área e, a partir disso, optou por utilizar dois questionários como instrumento de captação de dados e informações a respeito do projeto de implementação. Um, com terminologia técnica informática e com mais detalhes sobre o processo Gestão da Configuração, e outro, abordando aspetos gerais sobre a qualidade de serviço das TI. Mas ambos com o mesmo objetivo de investigar o papel que a GC pode exercer na melhoria dos processos de uma instituição.

Apesar de seu esforço, Esteves relata que não foi possível acompanhar toda a implementação de GC devido ao tempo limitado para finalizar a pesquisa. Além disso, afirma que não havia dados que servissem de indicadores de comparação entre um momento prévio e o momento final do trabalho, uma vez que a SRT não possuía um framework para a gestão de serviços de TI anteriormente. Entretanto, os pesquisadores garantem, com base no estudo feito, que os serviços de TI podem se beneficiar se as falhas detectadas durante a implantação forem corrigidas segundo as práticas ITIL.

Já no trabalho de Cabrita (2017), foi abordada a criação de um modelo de implementação da GC voltado para empresas de formação profissional. A motivação do trabalho foi baseada na necessidade de melhorar os serviços prestados e preparar a organização para obter certas certificações de mercado.

Ainda segundo o autor, houve dificuldades ao equalizar os níveis de conhecimento ITIL dos colaboradores da empresa e aproveitou da situação para sugerir que todos os envolvidos em um projeto como este devem possuir certificação ITIL v3 Foundation, a fim de facilitar o sincronismo do time envolvido. 
Apesar de o objetivo do artigo ter sido a implementação da GC em uma organização, Cabrita também afirmou que isso não foi possível devido ao horizonte temporal reduzido. Entretanto, foi possível a criação de um modelo específico de implantação da GC para empresas desse ramo.

Embora o tempo não seja o maior aliado dos trabalhos aqui citados, este artigo relata, com detalhes, uma implementação da GC completa e bem-sucedida, quantificando tal sucesso por meio de coletas de dados realizadas nos momentos de pré e pósimplantação deste trabalho.

\section{Metodologia}

Conforme já mencionado, o órgão público, objeto de estudo deste trabalho, já possui uma ferramenta CMDB de um dos maiores fornecedores de software da área, a qual possibilita descobertas de toda a arquitetura de TI, automatiza relacionamentos entre os CIs descobertos, provê relatório de análise de impacto, disponibiliza APIs para integrações com outras ferramentas, entre outras funções.

Outras ferramentas de monitoramento alimentam o CMDB com informações de estado de elementos de rede, servidores, serviços, softwares, computadores clientes e, inclusive, seus relacionamentos. Com isso, o CMDB se torna uma base federada e confiável, na qual devem ser criados manualmente apenas a camada superior dos serviços da organização, como: aplicações, funções e transações de negócio, serviços específicos de infraestrutura, entre outros CIs que não são descobertos automaticamente.

As atividades inerentes a este trabalho foram divididas em sete etapas, as quais foram definidas entre os profissionais envolvidos, tendo como objetivo facilitar o entendimento das responsabilidades e deixar bem definido o prazo especificado para cada tarefa.

\subsection{Coleta de Dados: estado inicial da Gestão de Configuração}

Com o propósito de captar a maturidade do módulo de Gestão de Configuração no órgão público, optou-se pela aplicação de um questionário e pela realização de entrevistas individuais com os coordenadores de cada área e gestores dos principais processos ITIL (Configuração, Mudança, Incidente, Problema, Nível de Serviço, Requisições e Conhecimento). O principal objetivo foi absorver o estado inicial dos principais processos que interagem com a GC, além de medir e mensurar os diferentes pontos de vista da camada gerencial do núcleo de TI da organização.

\subsection{Definição de Nível de Granularidade de CIs}

O termo 'Configuração' que tanto se fala neste trabalho é um termo genérico e isso não é por acaso. Um Item de Configuração (CI) refere-se a qualquer elemento que deve ser considerado no negócio para que ocorra a entrega de um serviço de TI, por exemplo: hardware, software, um documento PDF, uma VLAN do switch ou até mesmo um atributo de uma tabela do banco de dados [Ciesielska 2017].

O nível de granularidade que a Gestão de Configuração deve atingir depende diretamente da necessidade inicial do negócio. "Inicial" porque este nível pode ser aprimorado caso a organização opte em aprofunda-lo ou até mesmo torná-lo mais superficial. Com isso, para que este nível fosse definido de forma eficaz para as

isys: Revista Brasileira de Sistemas de Informação (iSys: Brazilian Journal of Information Systems) 
necessidades do negócio, uma reunião foi agendada junto ao gerente de projeto, consultor ITIL e demais partes interessadas do projeto.

\subsection{Validação de integrações entre ferramentas de monitoramento e CMDB}

A primeira tarefa prática é a verificação das integrações já mencionadas, por meio da comparação entre os CIs apresentados nas ferramentas alimentadoras e os CIs contidos no CMDB. O objetivo desta atividade é garantir a integridade dos dados que estão sendo transportados entre essas ferramentas.

Nesta etapa, devem ser tratados separadamente as ferramentas de monitoramento e o módulo de descoberta, os quais são os principais alimentadores de CIs no CMDB.

Em relação às ferramentas de monitoramento, cada área de TI do órgão ficou encarregada de manter sua parte do monitoramento atualizado, ou seja, monitores de bancos de dados foram de responsabilidade da Equipe de Banco de Dados, monitores de elementos de rede foram atualizados pela Equipe de Redes, e assim sucessivamente.

Similarmente, o módulo de descoberta do CMDB deve ser configurado corretamente para que seja possível alcançar toda a infraestrutura de TI do órgão público e retratar, com legitimidade, seus relacionamentos.

A descoberta do CMDB trabalha com duas possibilidades: acesso remoto via diversos protocolos de conexões (TCP, SMNP, RPC, LDAP, SSH, RDP, Telnet, entre outros) ou pode ser baseado em agente, que é um programa instalado nos computadores. Neste sentido, reuniões com cada área de TI foram registradas para definir a melhor estratégia de trabalho, alinhar pontos de vistas e expectativas, identificar e corrigir problemas durante o processo de descoberta de CIs.

\subsection{Configuração de Tempo de Descarte de CIs}

O universo de TI de uma organização é repleto de mudanças, independentemente do tipo de ambiente de produção, homologação, desenvolvimento ou teste. A Gestão de Configuração também precisa estar apta para resolver esta questão.

Para aumentar a confiabilidade dos dados apresentados no CMDB, existe um atributo em todo CI chamado tempo de descarte. Em cada tipo de CI, este atributo pode receber um valor numeral padrão, que é representado em dias. Este valor refere-se à quantidade de dias limite que o CI permanecerá na base de dados sem que seja acessado pelo monitoramento ou pela descoberta automatizada.

Durante duas reuniões semanais do projeto, este assunto foi abordado e discutido entre as partes interessadas. A principal preocupação do órgão teve relação com o intervalo de dias que um CI permaneceria no CMDB, mesmo que não esteja mais em uso no ambiente tecnológico.

\subsection{Modelagem do CMDB}

Nesta fase, a qual é baseada no nível de granularidade de CIs definido, a atenção da equipe deve ser elevada, uma vez que esta é uma tarefa predecessora de todas as atividades seguintes. Portanto, a fim de reduzir as chances de falhas, foi proposta aos gerentes do órgão a formação de uma equipe dedicada a esta atividade. Esta equipe recebeu a capacitação necessária para realizar as tarefas de modelagem na ferramenta CMDB. Além 
disso, apoio técnico também foi disponibilizado durante as atividades, a fim de garantir que as melhores práticas de modelagem de CIs, propostas pelo fornecedor da ferramenta CMDB [HPE Software 2015], fossem aplicadas.

Já que um conjunto de ferramentas é responsável por alimentar o CMDB federado da organização, cada uma das integrações foi revisada e, em alguns casos, corrigida ou melhorada. Consequentemente, a função de descoberta da ferramenta CMDB (sem agente) também passou por algumas validações e ajustes, visando capturar dados de inventário dos CIs de forma eficaz, evitando assim desperdício de recursos em descoberta de nós não relacionados à infraestrutura de serviços da organização [HPE Software, 2016].

Por isso, uma grande parte dos CIs do CMDB foram criados a partir da função de descoberta da ferramenta, que funciona da seguinte forma: credenciais de acesso foram cadastradas para diferentes tipos de protocolos [HPE Software 2016]. Alguns exemplos são os protocolos Telnet e SSH que são utilizados para disponibilizar acesso remoto a servidores Windows e/ou Linux. Dessa forma, o módulo de descoberta da ferramenta CMDB realiza diversas queries que retornam dados de inventário dos servidores. Estes dados são coletados e armazenados na ferramenta CMDB diariamente. O mesmo ocorre para roteadores, switches, sistemas de virtualização, entre outros.

\subsection{Avaliação das regras de Análise de Impacto}

Resumidamente, um relacionamento entre dois CIs possui determinado peso de impacto, o qual pode ser customizado a fim de retratar a real dependência entre um elemento e outro. Quanto mais próximo da realidade este impacto estiver do ambiente real, mais pontual será a informação retornada em um processo de mudança.

Dessa forma, a ferramenta CMDB permite prever o impacto de uma alteração no ambiente, mostrando quais CIs seriam afetados. Por exemplo, se uma mudança prevê a implantação de uma nova versão de um sistema de cadastro de clientes, o CMDB é capaz de alertar que o serviço de consulta de clientes será afetado por esta alteração.

Diante disso, existem três possibilidades referentes à gestão de Análise de Impacto. A primeira refere-se a manter o padrão da ferramenta CMDB, ou seja, nenhuma alteração seria feita a não ser que houvesse necessidade. A segunda proposta é parecida com a primeira: alterações no peso dos relacionamentos seriam realizadas apenas em relacionamentos específicos entre determinados tipos de CI. Na terceira, e mais trabalhosa, todos os relacionamentos e seus pesos deveriam ser revistos, a fim de garantir uma modelagem mais próxima possível da realidade.

Apesar de a terceira opção refletir um ambiente perfeito, a decisão de qual caminho seguir deve levar em consideração o custo e o benefício de cada uma das opções.

\subsection{Coleta de Dados: resultados pós-implantação}

Conforme relatado na seção de objetivos deste trabalho, entrevistas presenciais foram realizadas para absorver informações dos principais representantes dos usuários da GC: os gestores de processos ITIL e coordenadores das respectivas áreas de TI.

Durante as entrevistas, o mesmo questionário utilizado na pré-implantação foi reaplicado nesta etapa de pós-implantação, visando uma explícita comparação dos dados

isys: Revista Brasileira de Sistemas de Informação (isys: Brazilian Journal of Information Systems) 
entre as etapas. Além disso, discussões e comentários apresentados durante as entrevistas pelos profissionais da organização foram registrados em anotações.

Em suma, foi de grande importância capturar o nível de satisfação do usuário de TI com o novo módulo de GC implantado e, consequentemente, registrar sugestões de melhoria em processos da GC no órgão em estudo.

\subsection{Análise dos resultados obtidos}

Na área de Tecnologia da Informação, implantação de softwares ou serviços requerem um estudo prévio do ambiente no qual esta será realizada. E mais importante que o estudo prévio é a análise de valor agregado à ser entregue aos usuários diretos e indiretos.

Nesta etapa do trabalho, cabe a interpretação das informações dadas pelo corpo de coordenadores de TI e donos de processos ITIL, por meio da coleta de dados citada anteriormente. A partir das informações coletadas em entrevistas, foi possível obter informações abertas e qualitativas em relação ao projeto, deixando os questionários coletarem, principalmente, dados quantitativos para análises estatísticas.

\section{Resultados obtidos}

Nesta seção, estão dispostas as subseções referente às entrevistas e questionários realizados em dois momentos distintos: antes e após a implantação de GC.

\subsection{Coleta de dados: pré-implantação de GC}

Para esta primeira coleta de dados, um questionário com dezenove questões foi desenvolvido pelo consultor ITIL deste projeto para ser aplicado durante as entrevistas, nas quais participaram 16 funcionários, entre coordenadores e donos de processos ITIL do órgão público. Na Tabela 2, encontra-se o resultado consolidado. No contexto das entrevistas: um evento representa um incidente, requisição, problema ou mudança.

Como parte dos resultados colhidos, foram selecionados alguns comentários de entrevistados conforme suas reincidências.

Apesar do resultado consolidado da questão 16, da Tabela 2, 68\% dos entrevistados manifestam que as alterações em CIs possuem frequência elevada e não são replicadas para o $\mathrm{CMDB}$ com a devida prioridade. Isso dificulta a tratativa de falhas no ambiente, controle de mudanças e administração correta dos acordos internos e externos.

Outro caso abordado por $31 \%$ dos entrevistados relaciona os CIs como elementos conhecimentos e inseridos no $\mathrm{CMDB}$, porém havia dificuldades em gerir e realizar análises básicas devido à falta de confiança na base federada da organização.

Em suma, através de todo este processo inicial de captação de informações, foi possível definir o nível de maturidade, do órgão público em questão, em cada módulo ITIL. Dessa forma, a Tabela 3 representa os níveis de maturidade mensurados para os módulos de configuração e principais relacionados. 
Tabela 2. Resultado de questionário objetivo aplicado (pré-implantação)

\begin{tabular}{|c|c|c|}
\hline Pergunta & Sim & Não \\
\hline $\begin{array}{l}\text { 01. O conceito sobre o que são os serviços de TI é de conhecimento comum } \\
\text { (conceito ITIL)? }\end{array}$ & $31,25 \%$ & $68,75 \%$ \\
\hline 02. Existe um portfólio de serviços constantemente gerenciado? & $31,25 \%$ & $68,75 \%$ \\
\hline 03. Existe um catálogo de serviços claro para os usuários? & $31,25 \%$ & $68,75 \%$ \\
\hline 04. Existe um catálogo de serviços claro para os técnicos das áreas de TI? & $68,75 \%$ & $31,25 \%$ \\
\hline 05. Existe uma matriz de responsabilidade para cada serviço de TI? & $31,25 \%$ & $68,75 \%$ \\
\hline 06. Existe análise ou monitoramento sobre a qualidade dos serviços? & $6,25 \%$ & $93,75 \%$ \\
\hline 07. O modelo de categorização de eventos é $100 \%$ eficiente? & $25,00 \%$ & $75,00 \%$ \\
\hline $\begin{array}{l}\text { 08. O conceito de controle dos ativos de serviço é formal e de conhecimento } \\
\text { comum? }\end{array}$ & $31,25 \%$ & $68,75 \%$ \\
\hline $\begin{array}{l}\text { 09. As possíveis variações de demanda estão previstas e podem ser } \\
\text { controladas? }\end{array}$ & $12,50 \%$ & $87,50 \%$ \\
\hline $\begin{array}{l}\text { 10. Existe uma metodologia para gerenciamento de risco a gestão dos } \\
\text { serviços? }\end{array}$ & $0,00 \%$ & $100,00 \%$ \\
\hline 11. Existe um plano de tratativa para missão crítica? & $6,25 \%$ & $93,75 \%$ \\
\hline $\begin{array}{l}\text { 12. Os detalhes sobre a infraestrutura são conhecidos por todos } \\
\text { (relacionamento entre ICs)? }\end{array}$ & $43,75 \%$ & $50,00 \%$ \\
\hline 13. Existe um sistema eficiente para gerenciamento de mudanças? & $6,25 \%$ & $93,75 \%$ \\
\hline 14. Existe base de conhecimento ativa e gerenciada? & $75,00 \%$ & $25,00 \%$ \\
\hline $\begin{array}{l}\text { 15. Existe um sistema eficiente para gestão de configuração e ativos de } \\
\text { serviço da TI? }\end{array}$ & $25,00 \%$ & $75,00 \%$ \\
\hline 16. Existe uma CMDB ativa e atualizada? & $87,50 \%$ & $12,50 \%$ \\
\hline 17. É possível controlar a quantidade de mudanças normais e emergenciais? & $43,75 \%$ & $56,25 \%$ \\
\hline $\begin{array}{l}\text { 18. Existe uma metodologia e ferramentas para monitoramento dos ICs e } \\
\text { serviços? }\end{array}$ & $68,75 \%$ & $31,25 \%$ \\
\hline 19. Existem reuniões periódicas de Comitê Consultivo de Mudança (CCM)? & $12,50 \%$ & $87,50 \%$ \\
\hline
\end{tabular}

Tabela 3. Nível de maturidade de módulos ITIL no órgão (pré-implantação)

\begin{tabular}{|l|c|}
\hline Módulo ITIL & Nível de Maturidade (0 a 5) \\
\hline Configuração & 1,07 \\
\hline Mudança & 0,66 \\
\hline Requisição & 0,69 \\
\hline Problema & 0,63 \\
\hline Incidente & 0,58 \\
\hline Nível de Serviço & 0,48 \\
\hline
\end{tabular}




\subsection{Definição do Nível de Granularidade de CIs}

Durante uma reunião entre gerente de projeto, consultor ITIL e demais partes interessadas, a granularidade de CIs foi definida baseada na seguinte segmentação: categoria de aplicação, aplicação, URI de conexão, camada (aplicação, banco de dados, webservice, etc), nó servidor, informações de inventário, nome de cluster, nó de cluster, nó de rede, nó firewall. Ou seja, informações de inventário foram consideradas como o nível mais profundo da granularidade de CIs.

Um dos principais fatores que contribuíram para a definição do nível de granularidade de CIs foi a documentação de melhores práticas do fornecedor da ferramenta CMDB (HPE Software, 2015). Além disso, utilizou-se das entrevistas realizadas para absorver dos usuários as necessidades iniciais do negócio.

\subsection{Validação de integrações entre ferramentas de monitoramento e CMDB}

O universo de TI da organização é composto por cerca de mil e duzentos servidores (físicos ou virtuais), três mil e duzentos ativos gerenciáveis de rede (roteadores, switches e pontos de acesso), vinte e seis mil desktops e notebooks, oito mil e quinhentas impressoras gerenciáveis, entre outros tipos de CI que devem estar sob a Gestão de Configuração do modelo ITIL.

Para que o CMDB seja capaz de representar todo este universo de CIs, com legitimidade, é necessário o auxílio de outras ferramentas, as quais dominam cada uma das áreas da TI. Por exemplo: a ferramenta de gerenciamento de clientes finais (Endpoint Management) é utilizada para alimentar a base do CMDB com tais dados. Já a ferramenta de monitoramento de ativos de rede é utilizada para alimentar outra parte da base. Com isso, cada ferramenta auxiliar deve alimentar o CMDB de acordo com sua especialidade.

Neste caso, todo o universo de TI do órgão público foi dividido em dois grupos: o primeiro é composto pelos elementos de infraestrutura (servidores, aplicações, roteadores, switches, links, entre outros) e o segundo é composto pelo restante dos CIs (desktops, notebooks, impressoras, terminais de consulta).

Para o primeiro grupo de CIs, utilizou-se o módulo de descoberta do CMDB apenas via acesso remoto, ou seja, sem agente. Esta funcionalidade permitiu a unificação da gestão de descoberta dos CIs.

Em relação ao segundo grupo de CIs, existem integrações com uma ferramenta de inventário de clientes finais (desktops, notebooks e celulares) e com um banco de dados de inventário de equipamentos do órgão (impressoras, terminais de consulta, monitores).

\subsection{Configuração de Tempo de Descarte de CIs}

A quantidade de dias que um CI deve se manter na base de dados do CMDB, mesmo que este já não exista mais fisicamente, depende de alguns fatores importantes que não devem ser generalizados para quaisquer tipos de CI. Os desktops e CIs do negócio, por exemplo, possuem tempo de descarte nulo, ou seja, nunca serão deletados da base automaticamente, uma vez que é interessante existir o histórico de mudanças ocorridas desde sua criação no CMDB (alteração do atributo de memória física, tamanho de disco rígido).

Contudo, as partes interessadas deste projeto definiram que 40 dias seria o valor padrão para o atributo de tempo de descarte dos demais tipos de CI. Todavia, de acordo

isys: Revista Brasileira de Sistemas de Informação (isys: Brazilian Journal of Information Systems) 
com a maturidade da organização em relação a GC, este atributo poderá ser reduzido no futuro.

\subsection{Modelagem do CMDB}

Nesta etapa de modelagem de CIs, no ambiente do órgão, foram considerados dois grupos de CIs.

O primeiro grupo refere-se aos CIs que são inseridos no CMDB automaticamente pela função de descoberta da ferramenta [HPE Software 2016]. Alguns exemplos principais são: roteadores e switches, servidores e seus dados (se físicos ou virtuais; nome, informações de rede, sistemas de arquivos, interfaces de rede), softwares (banco de dados, JBOSS, Apache, Tomcat), plataformas de serviços via web (web service SOAP, REST), serviços de alta disponibilidade (Failover Clustering), serviços de gerenciamento de rede (Controlador de Domínio, DHCP, DNS), protocolos de acesso remoto (SSH, Telnet, FTP, $R P C)$ [Cabrita 2017]. Cada CI descoberto tem seus relacionamentos mapeados automaticamente pela função do CMDB, por exemplo, os serviços de gerenciamento de rede serão relacionados ao respectivo servidor onde foi detectado. A mesma situação ocorre para softwares, serviços via web, entre vários outros.

Já o segundo grupo, que não é descoberto como o primeiro, representa CIs de negócio da organização. Alguns exemplos são: nomes de aplicações e sistemas, serviços providos por essas aplicações, nomes das principais transações web de uma aplicação (para fins de monitoramento), pontos de conexão (seja para acesso a banco de dados, aplicação web ou web services). A princípio, os CIs deste grupo devem ser relacionados manualmente aos CIs do primeiro grupo, formando relações diretas ou indiretas entre os elementos de negócio e os elementos de infraestrutura da organização.

A Figura 2 representa a modelagem utilizada para o relacionamento de CIs de ambos os grupos. Entende-se como modelagem a padronização dos relacionamentos entre diferentes tipos de CI. Tal modelo foi desenvolvido baseado em documentação de melhores práticas do fornecedor da ferramenta CMDB em questão [HPE Software 2015]. 


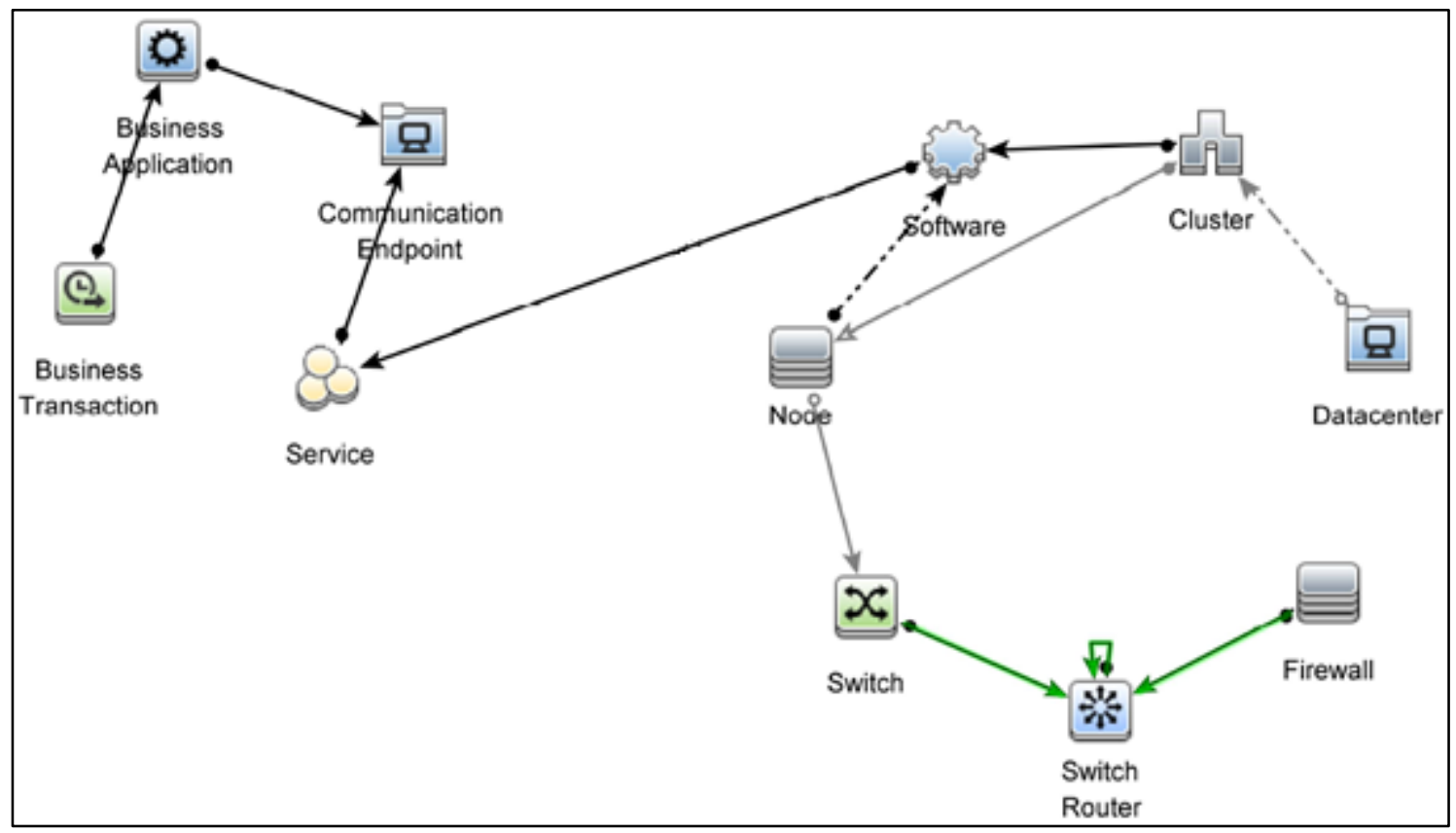

Figura 2. Modelagem desenvolvida para representar a infraestrutura tecnológica da empresa.

\subsection{Avaliação das Regras de Análise de Impacto}

As regras de análise de impacto são responsáveis, principalmente, por facilitar a identificação de sistemas afetados frente a ocorrência de eventos. Por este motivo, é importante definir bem como estas regras serão configuradas, de forma que os relacionamentos entre CIs transpareçam suas composições e dependências.

Neste estudo de caso, verificou-se que a ferramenta CMDB possui regras prédefinidas em sua configuração padrão. Além disso, tais regras estavam de acordo com a necessidade do negócio. Portanto, as partes interessadas do projeto definiram que, inicialmente, elas seriam mantidas e utilizadas para representar os relacionamentos entre CIs do ambiente.

Com o objetivo de exemplificar um cenário simples de utilização da Análise de Impacto, supõe-se que um evento de mudança para alteração de configurações de capacidade de um serviço web foi criado e aprovado. Por meio das regras de análise de impacto configuradas no CMDB, a gerência de TI é capaz de identificar qual o sistema afetado por esta mudança, uma vez que o serviço web está vinculado a um host está relacionado a uma aplicação.

A Figura 3 representa o cenário descrito acima, possibilitando melhor entendimento de como o impacto é calculado. Neste cenário, o CI Apache Tomcat A é o CI afetado - seja por manutenção programada, atualização, reinicialização ou qualquer outro motivo. Porém, uma vez que este possui um relacionamento de dependência com o CI Aplicacao Depox (PRD), o CMDB projeta tal impacto para facilitar a sua visualização antes que a mesma ocorra. Esta funcionalidade, normalmente, é utilizada para identificar os impactos de uma mudança futura em um ambiente de TI inter-relacionado. 


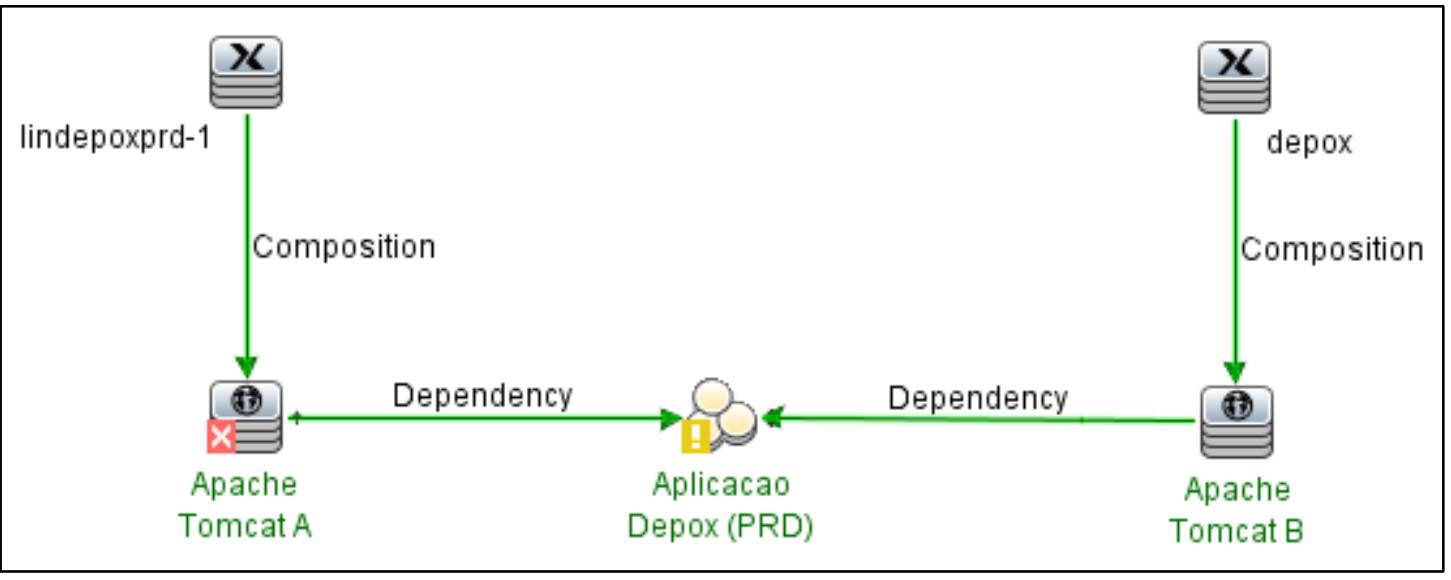

Figura 3. Simulação de análise de risco utilizando a ferramenta CMDB.

\subsection{Coleta de dados: pós-implantação de GC}

Conforme mencionado neste trabalho, não é interessante que a ITIL, em especial a GC, seja conhecida dentro de uma empresa apenas por uma pequena parte da organização (diretoria, analistas de configuração e/ou profissionais implementadores). Isso porque os benefícios gerados por seu funcionamento dependem do nível de abrangência que o módulo atinge dentro de uma organização [Yamamoto 2017]. No caso deste trabalho, é de extrema importância que os usuários de TI conheçam e tenham domínio sobre a configuração dos ICs e como eles se relacionam e possibilitam a prestação dos serviços de TI da organização.

Em consequência disso, além deste projeto ter sido apoiado pela alta gerência da organização, os usuários de TI também foram contextualizados sobre as funções da Gestão de Configuração dentro da organização em que trabalham.

Levando em consideração estes aspectos, uma nova coleta de dados foi realizada após seis meses da implantação da Gestão de Configuração, utilizando apenas o formato de questionário. Como parte da coleta, foi disponibilizado um espaço para descrição de observações e sugestões pertinentes à GC e outros módulos relacionados.

É importante registrar que após a conclusão da implantação da GC, os módulos de Mudança e Problema também começaram a ser trabalhados no órgão público. Com isso, os dados obtidos nesta fase foram, de certa forma, influenciados por eles. Considerando estas observações, a Tabela 4 retrata o resultado sintético da coleta realizada, a qual se baseou nas mesmas perguntas utilizadas na primeira etapa de coleta de dados. 
Tabela 4. Resultado de questionário objetivo aplicado (pós-implantação)

\begin{tabular}{|c|c|c|}
\hline Pergunta & Sim & Não \\
\hline $\begin{array}{l}\text { 01. O conceito sobre o que são os serviços de TI é de conhecimento comum } \\
\text { (conceito ITIL)? }\end{array}$ & $75,00 \%$ & $25,00 \%$ \\
\hline 02. Existe um portfólio de serviços constantemente gerenciado? & $18,75 \%$ & $81,25 \%$ \\
\hline 03. Existe um catálogo de serviços claro para os usuários? & $62,50 \%$ & $37,50 \%$ \\
\hline 04. Existe um catálogo de serviços claro para os técnicos das áreas de TI? & $50,00 \%$ & $50,00 \%$ \\
\hline 05. Existe uma matriz de responsabilidade para cada serviço de TI? & $37,50 \%$ & $62,50 \%$ \\
\hline 06. Existe análise sobre a qualidade dos serviços? & $43,75 \%$ & $56,25 \%$ \\
\hline 07. O modelo de categorização de eventos é $100 \%$ eficiente? & $6,25 \%$ & $93,75 \%$ \\
\hline $\begin{array}{l}\text { 08. O conceito de controle dos ativos de serviço é formal e de conhecimento } \\
\text { comum? }\end{array}$ & $31,25 \%$ & $68,75 \%$ \\
\hline $\begin{array}{l}\text { 09. As possíveis variações de demanda estão previstas e podem ser } \\
\text { controladas? }\end{array}$ & $25,00 \%$ & $75,00 \%$ \\
\hline $\begin{array}{l}\text { 10. Existe uma metodologia para gerenciamento de risco a gestão dos } \\
\text { serviços? }\end{array}$ & $25,00 \%$ & $75,00 \%$ \\
\hline 11. Existe um plano de tratativa para missão crítica? & $6,25 \%$ & $93,75 \%$ \\
\hline $\begin{array}{l}\text { 12. Os detalhes sobre a infraestrutura são conhecidos por todos } \\
\text { (relacionamento entre ICs)? }\end{array}$ & $43,75 \%$ & $56,25 \%$ \\
\hline 13. Existe um sistema eficiente para gerenciamento de mudanças? & $75,00 \%$ & $25,00 \%$ \\
\hline 14. Existe base de conhecimento ativa e gerenciada? & $56,25 \%$ & $43,75 \%$ \\
\hline $\begin{array}{l}\text { 15. Existe um sistema eficiente para gestão de configuração e ativos de } \\
\text { serviço da TI? }\end{array}$ & $75,00 \%$ & $25,00 \%$ \\
\hline 16. Existe uma CMDB ativa e atualizada? & $81,25 \%$ & $18,75 \%$ \\
\hline 17. É possível controlar a quantidade de mudanças normais e emergenciais? & $100,00 \%$ & $0,00 \%$ \\
\hline $\begin{array}{l}\text { 18. Existe uma metodologia e ferramentas para monitoramento dos ICs e } \\
\text { serviços? }\end{array}$ & $56,25 \%$ & $43,75 \%$ \\
\hline 19. Existem reuniões periódicas de Comitê Consultivo de Mudança (CCM)? & $100,00 \%$ & $0,00 \%$ \\
\hline
\end{tabular}

Baseado nos dados coletados, a Figura 4 representa um resultado comparativo entre os resultados colhidos na pré e pós-implantação de GC, avaliando apenas as respostas de valor 'sim'. Desta maneira, foi possível constatar com clareza os itens que evoluíram do ponto de vista do usuário final de TI. 
Existem reuniões periódicas do CCM?

Existe uma metodologia e ferramentas para.

É possível controlar a quantidade de mudanças.

Existe uma CMDB ativa e atualizada?

Existe um sistema eficiente para gestão de.

Existe base de conhecimento ativa e gerenciada?

Existe um sistema eficiente para gerenciamento.

Os detalhes sobre a infraestrutura são conhecidos.

Existe um plano de tratativa para missão crítica?

Existe uma metodologia para gerenciamento de.

As possíveis variações de demanda estão previstas.

O conceito de controle dos ativos de serviço é.

O modelo de categorização de eventos é eficiente.

Existe análise ou monitoramento sobre a.

Existe uma matriz de responsabilidade para cada.

Existe um catálogo de serviços claro para os.

Existe um catálogo de serviços claro para os.

Existe um portfólio de serviços constantemente.

O conceito sobre o que são os serviços de TI é de.

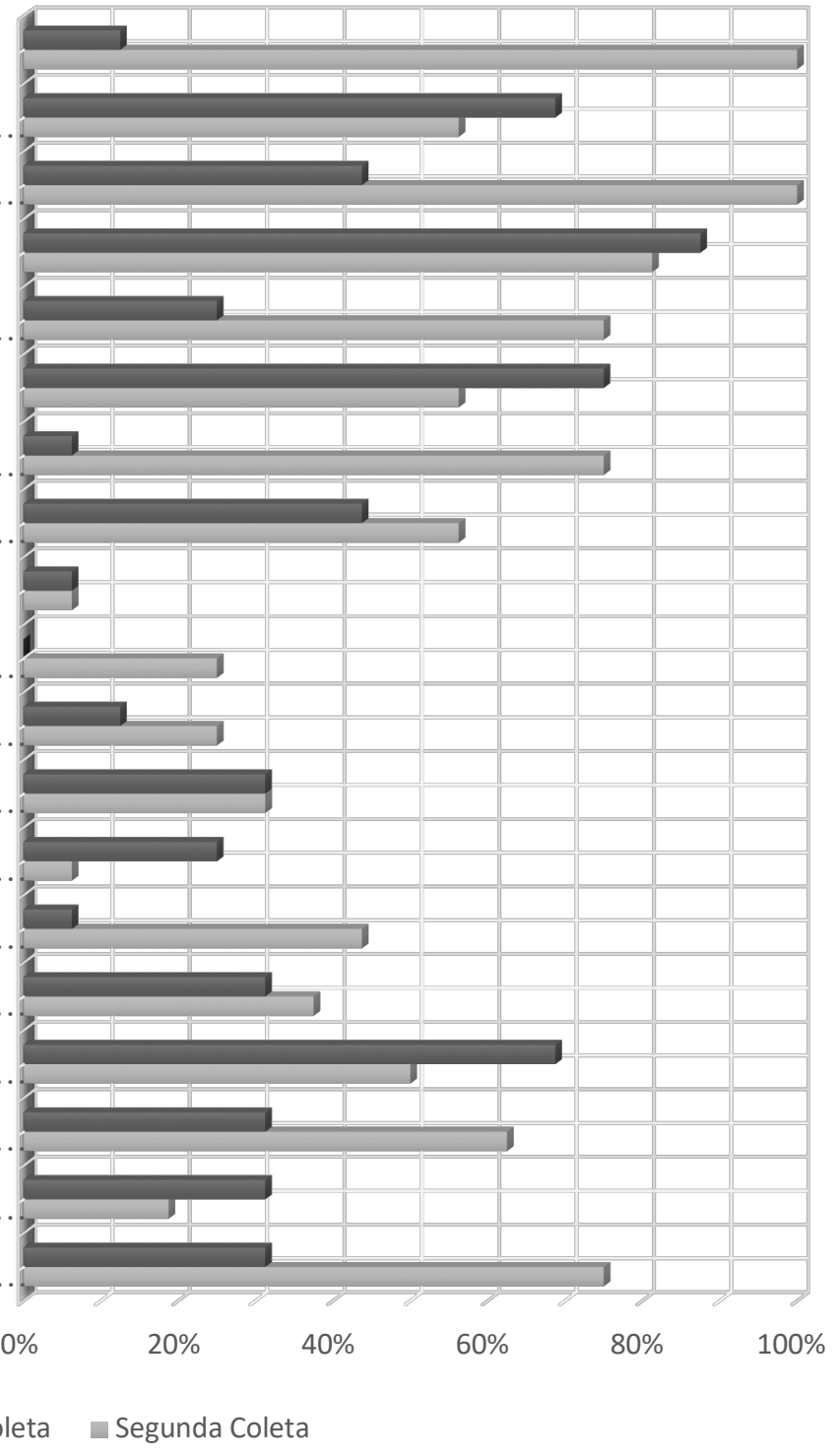

- Primeira Coleta $\square$ Segunda Coleta

Figura 4. Comparativo dos dados coletados nas etapas de pré e pós-implantação de GC.

\section{Análise de Resultados}

Desde o planejamento do projeto descrito neste trabalho, a diretoria do órgão público foi diretamente envolvida e ativa nas decisões tomadas, sendo este o principal fator para $o$ sucesso do trabalho. Além disso, os coordenadores de áreas entenderam a necessidade de novos processos às atividades de TI que o órgão suporta, a fim de que o conhecimento seja documentado, os riscos reduzidos e as alterações no ambiente sejam controladas e conhecidas.

Contudo, foi possível identificar certa resistência na aceitação dos novos processos por alguns analistas de TI de diferentes áreas como: desenvolvimento, banco de dados, aplicação, operação e suporte. Entre os processos que mais geraram resistência está o processo de atualização constante do CMDB. Para que isso fosse possível, a 
organização também decidiu investir no módulo de Gestão de Mudança (GM), com o objetivo de impedir que alterações no ambiente de TI fossem concluídas sem serem replicadas ao CMDB.

Já em relação aos dados coletados, é possível afirmar que uma implantação do módulo de GC, do framework ITIL, não está concluída apenas pelo fato de as ferramentas estarem instaladas e implementadas de acordo com as melhores práticas do fornecedor. Por este motivo, é extremamente importante a divulgação do projeto e a capacitação básica das partes interessadas, para que haja consentimento e entendimento dos novos processos a serem implementados no ambiente tecnológico da empresa. Tais ações poderiam gerar melhorias consideráveis no momento da segunda coleta de dados realizada, por exemplo, no resultado das perguntas relacionadas ao relacionamento de ICs e à ferramenta CMDB (conforme Figura 5).

De qualquer forma, foi possível notar melhorias consideráveis neste assunto, exceto em relação à primeira pergunta da Figura 5, onde a porcentagem de respostas de valor 'sim' reduziu de $87,5 \%$, na primeira coleta, para $81,3 \%$, na segunda coleta. Não foi possível identificar o motivo desta redução, mas uma possível justificativa reside no fato que os usuários adquiriram maior entendimento de Gestão de Configuração, se comparado com o momento da primeira coleta de dados. Consequentemente, nesta segunda coleta, as perguntas foram respondidas com maior precisão e domínio do assunto.

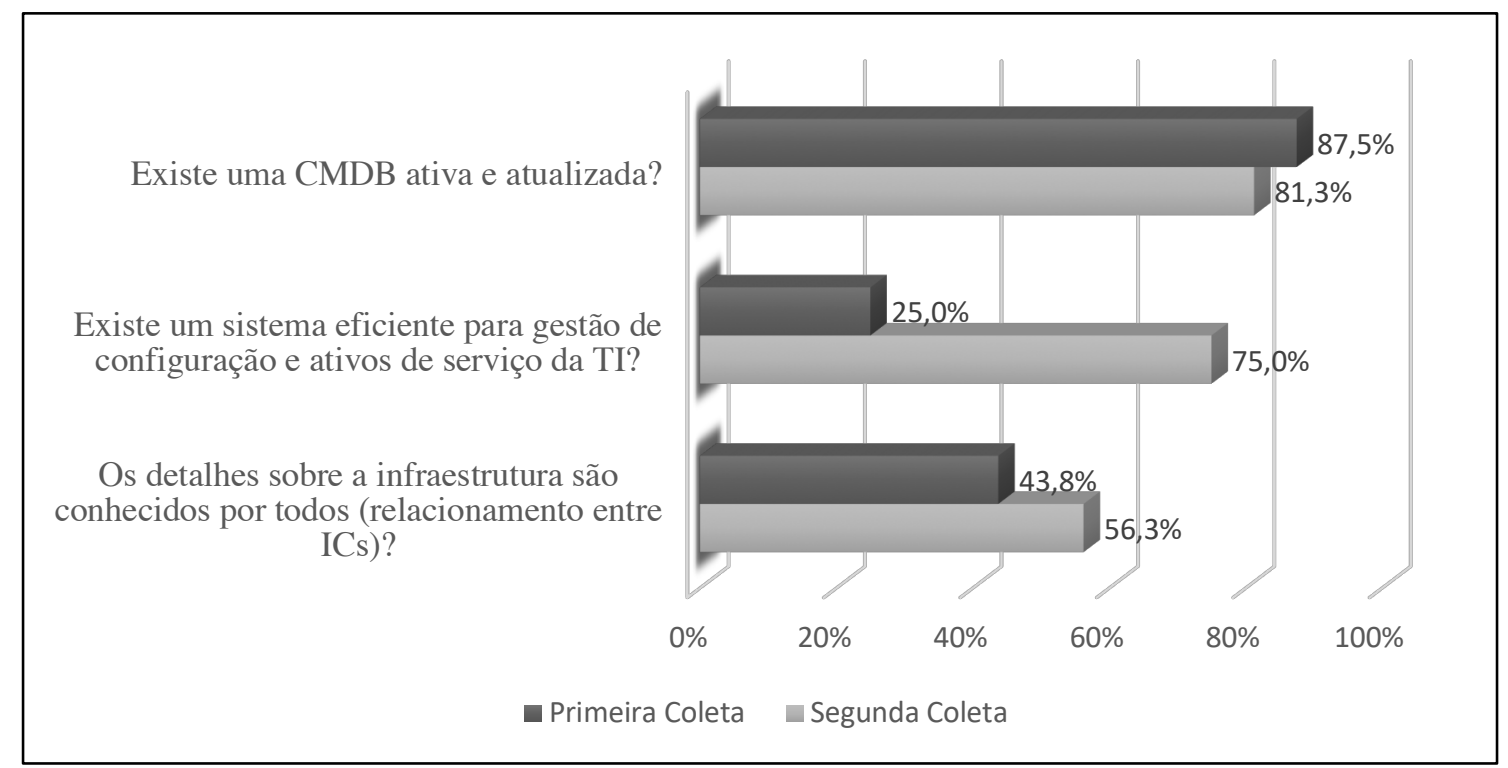

Figura 5. Comparativo dos dados coletados: perguntas com relação direta ao CMDB.

Conforme já relatado neste trabalho, outros módulos da ITIL são suportados pela Gestão de Configuração. De fato, baseado no comparativo dos dados coletados, pôde-se notar que a maturidade da organização frente à Gestão de Mudança foi a mais beneficiada, tendo como parâmetros outros módulos relacionados à GC. No ambiente de TI do órgão em estudo, a Figura 6 demonstra esta situação de forma explícita, além de confirmar uma dependência considerável da GM em relação à GC. 


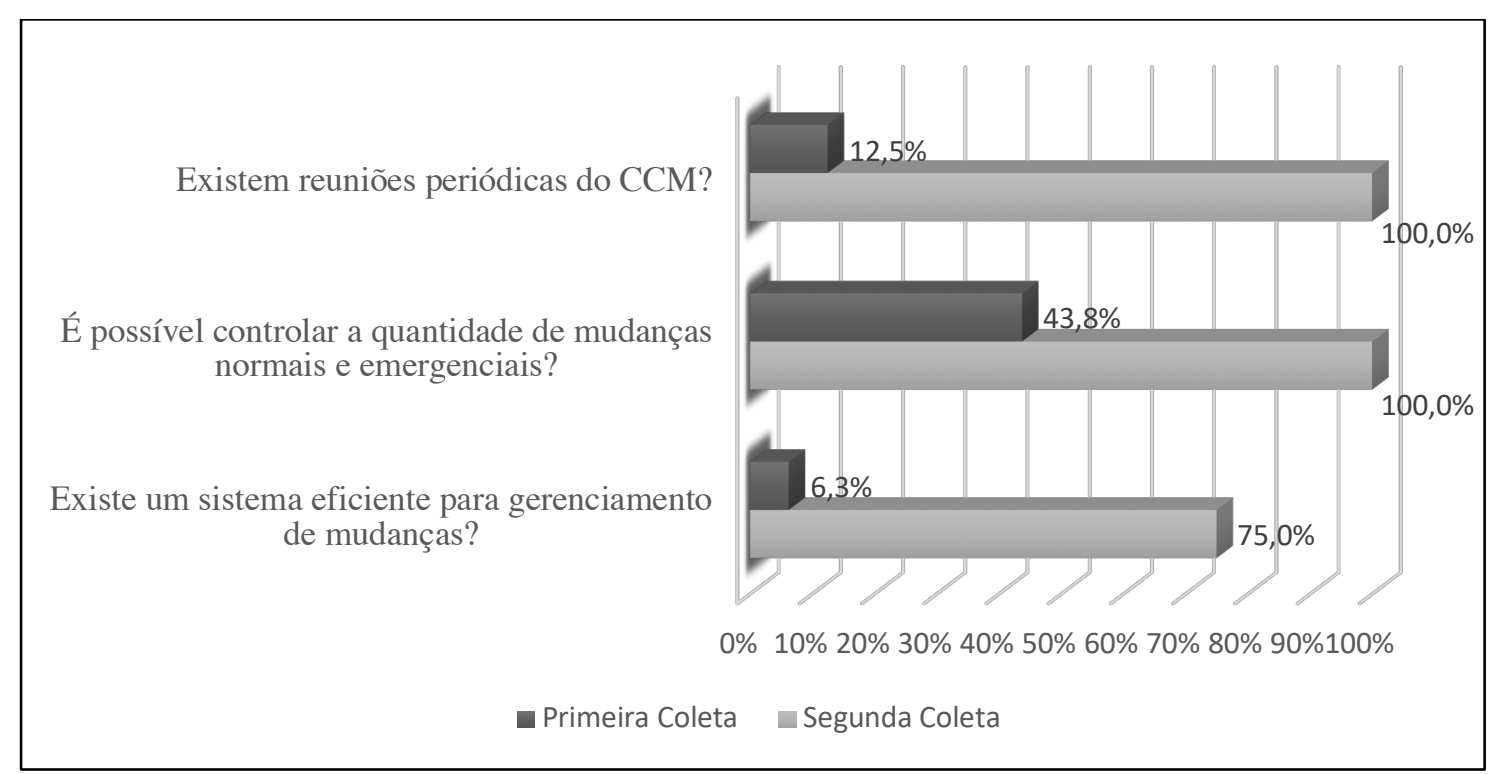

Figura 6. Comparativo dos dados coletados: perguntas com relação direta ao módulo de Gestão de Mudanças (ITIL).

\section{Conclusões}

A falta de gerenciamento de uma grande estrutura de TI implica em uma série de riscos à qualidade do serviço prestado. Por esse motivo, este trabalho teve por objetivo a implantação do módulo de Gestão de Configuração do framework ITIL em uma empresa brasileira de grande porte. Para que isso fosse possível, contou com vários fatores, dentre os quais se destacam: o apoio gerencial empregado ao projeto, equipe de profissionais especializada e por ter uma base bem definida em documentações de melhores práticas do mercado (ITIL e fornecedor CMDB).

Como resultado obtido através deste estudo, pode-se notar a importância da GC em meio ao gerenciamento de serviços de TI. Além disso, foi possível perceber que a GC tem grande influência em outros módulos do framework, em especial: a Gestão de Mudanças. Outro ponto importante descrito neste artigo tem relação com a melhoria contínua dos processos recentemente implantados, principalmente no que se refere à divulgação e capacitação básica dos colaboradores envolvidos.

Tendo em consideração os resultados obtidos, o intervalo de tempo entre a primeira e a segunda coleta de dados, cerca de 6 meses, foi relativamente curto para que os usuários de TI da organização absorvessem completamente os benefícios da GC. De fato, para trabalhos futuros, sugere-se que, para que os resultados sejam refletidos entre os colaboradores, este intervalo de tempo seja discutido com as partes interessadas, levando em consideração parâmetros como o tamanho da organização e/ou da área de TI, cultura da empresa e nível de apoio das áreas interessadas. 


\section{Referências}

Axelos Website (2018). "What is ITIL Best Practice?", https://www.axelos.com/bestpractice-solutions/itil/what-is-itil, Setembro.

Batmunkh, T., Küçük, B. e Oztürk, Muhammet A. (2014). "ITIL v3 and ITIL 2011. The changed process and the reason of change", https://torashiroi.wordpress.com/2014/05/10/itil-v-3-and-itil-2011-the-changedprocess-and-the-reason-of-change, Setembro.

Bartusevics, A. e Novickis, L. (2015). Models for Implementation of Software Configuration Management. Procedia Computer Science, 43:3-10.

Bayona, S., Baca, Y. e Vela, G. (2017). "Gestión de servicios de TI usando ITIL v3: caso de estudio", In Proceedings of the 12th Iberian Conference on Information Systems and Technologies, pages 1-6. IEEE.

Cabrita, M. (2017). "ITIL v.3 - Information Technology Infrastructure Library: Gestão da Configuração Aplicação em Empresas de Formação Profissional”. (Master's thesis) Barcarena, Atlântica - Escola Universitária de Ciências Empresariais, Saúde, Tecnologias e Engenharia, Julho.

Ciesielska, M. (2017). Implementation of ITIL Service Lifecycle in small and mediumsized enterprises of Polish ICT sector. Information Systems in Management, 6: 85-96.

Conger, S., Winniford, M., e Erickson-harris, L. (2008). Service management in operations, In AMCIS 2008 Proceedings, p. 362, http://aisel.aisnet.org/amcis2008/362, Outubro.

Esteves, R. (2012). "Implementação do Processo de Gestão de Configuração do framework ITIL - um Estudo de Caso". Dissertação (mestrado). Instituto Politécnico de Bragança, Escola Superior de Tecnologia e de Gestão, Bragança.

HPE Software (2015). "HP Best Practices: Service Modeling - Software Version: 3.00", https://goo.g1/7A2ubn, Dezembro.

HPE Software (2016). "Universal uCMDB: Discovery and Integrations Content Guide Discovery Activities", $\quad$ http://cmshelpcenter.saas.hp.com/CMS/10.30/cpdocs/docs/eng/pdfs/HPE_UCMDB_DICG_Discovery_Activities.pdf, Outubro.

HPE Software Website (2016). “Assisted Modeling”. http://cmshelpcenter.saas.hp.com/CMS/ucmdbbrowser/4.12/Content/Browser/Assiste dModeling.htm, Dezembro.

HPE Software Website (2016). "Service Modeling". http://cmshelpcenter.saas.hp.com/CMS/ucmdbbrowser/4.12/Content/Browser/Servic eModeling.htm, Dezembro.

Iden, J. e Eikebrokk, T. (2013). Implementing IT Service Management: A systematic literature review. International Journal of Information Management, 33(3):512-523.

Jiang, K. e Kamali, R. (2008). Integration of Configuration Management into the IT curriculum, In Proceedings of the 9th ACM SIGITE conference on Information technology education, pages 183-186. ACM 
Lema, L., Calvo-Manzano, J., Colomo-Palacios, R. e Arcilla, M. (2015). ITIL in small to medium-sized enterprises software companies towards an implementation sequence. Journal of software: evolution and process, 27(8):528-538.

Marquis, H. (2007). ITIL And The Evolving CMDB. Business Communications Review, 37(2):54-57.

Marrone, M. e Kolbe, L. (2011). Impact of IT Service Management Frameworks on the IT Organization. Business \& Information Systems Engineering, 3(1):5-18.

Mora, M., Raisinghani, M., O'Connor, R., Gomez, J. e Gelman, O. (2014). An extensive review of IT service design in seven international ITSM processes frameworks: Part I. International Journal of Information Technology System Appoach (IJITSA), 7(2):83107.

Orta, E., Ruiz, M., Hurtado, N. e Gawn, D. (2014). "Decision-making in IT Service Management: a simulation based approach”. Decision Support Systems, 66:36-51.

Palma, F. (2013). "Utilidade e garantia ao longo do ciclo de vida do serviço de TI". http://www.portalgsti.com.br/2013/09/utilidade-garantia-napratica.html, Dezembro.

Yamada, H., Yada, T. e Nomura, H. (2013). Developing network Configuration Management Database system and its application - Data federation for network management. Telecommunication Systems, 52(2):993-1000.

Yamamoto, S. (2017). A Continuous approach to improve IT management. Procedia Computer Science, 121:27-35. 